\title{
大别-苏鲁造山带超高压榴辉岩和脉体磷灰石含氯特征 与变质流体演化
}

\author{
刘景波", 张灵敏，陈意，郭顺，程南飞 \\ 中国科学院地质与地球物理研究所, 岩石圈演化国家重点实验室, 北京 100029 \\ * 联系人, E-mail: jingboliu @ mail.igcas.ac.cn \\ 2013-05-26 收稿, 2013-06-07 接受 2013-07-29 网络版发表 \\ 国家重点基础研究发展计划(2009CB825001)和国家自然科学基金(41072049, 41090371)资助
}

\begin{abstract}
摘要 根据磷灰石中的氯组分含量, 研究了大别-苏鲁超高压变质带中榴辉岩和其中的脉体形 成过程中流体的盐度及其变化. 仰口榴辉岩中磷灰石在进变质过程中显示为氯含量增加, 反 映出俯冲的大陆岩石经受了一个逐步干化和流体活动性降低的过程; 在退变质过程中氯含量 降低, 反映超高压岩石折返过程中流体含量增加. 大别-苏鲁超高压变质带一些典型的露头, 包括池庄、青龙山、毛北、碧溪岭、毛屋、朱家冲和花凉亭等地的超高压榴辉岩及其中的脉 体, 其磷灰石成分变化很大, 指示榴辉岩相变质和脉体形成过程中流体盐度变化很大, 从低 盐度流体到高盐度流体均存在, 不同露头有所差异. 总体来看, 不同盐度的流体在变质过程 中普遍存在, 流体盐度的变化可能与大陆地壳俯冲/折返过程中岩石水化和脱水反应有关.
\end{abstract}

\section{关键词}

大别-苏鲁超高压 变质带

榴辉岩

磷灰石

变质流体

变质作用
高压-超高压变质岩石中, 矿物中的盐水包裹体 是一种最常见的类型 ${ }^{[1 ~ 11]}$. 这些盐水包裹体主要出现 在蓝晶石、绿辉石和石榴石等榴辉岩相矿物中，表明 它们出现在高压-超高压变质阶段. 目前含盐流体对 变质岩石成因影响的研究并不多, 很可能过低估计了 它们在变质过程中的作用 ${ }^{[12]}$, 已有的相平衡研究也 很少考虑这样的流体体系. 盐流体除流体包裹体的 记录外, 几乎忽略了其对应的矿物成分记录. 换言之, 哪些矿物记录了盐流体存在的信息? 其成分变化又 是如何与流体成分变化相关联的? 通过对大别-苏鲁 超高压变质带榴辉岩和脉体中磷灰石的氯含量进行 研究, 我们确定了高压-超高压变质过程中流体的盐 度变化. 根据磷灰石的氯含量变化, 可对大陆岩片俯 冲和折返过程中流体含量的变化特征予以制约.

\section{1 仰口榴辉岩中的磷灰石}

苏鲁造山带仰口榴辉岩是一个研究程度很高的
露头. 该榴辉岩体由变质反应边辉长岩、反应边榴辉 岩、细粒榴辉岩和粗粒榴辉岩(正常榴辉岩)组成, 显 示了明显的进变质特征. 变质反应边辉长岩仍然保 留早期岩浆结晶矿物, 如单斜辉石、斜方辉石和黑云 母等; 反应边榴辉岩和正常榴辉岩中早期岩浆矿物 已完全消失. 反应边榴辉岩中石榴石反应边发育, 正 常榴辉岩中反应边石榴石消失, 岩石转变为粒状变 晶结构. 反应边榴辉岩的温压估算指示出它们形成 于高压变质阶段, 并在超高压变质阶段有明显的再 平衡发生. 正常榴辉岩的温压估算则表明, 这些榴辉 岩在超高压条件下发生了完全重结晶. 我们对 3 类岩 石中的磷灰石进行了系统的成分研究, 结果显示它 们在 $\mathrm{OH}, \mathrm{Cl}$ 和 $\mathrm{F}$ 含量上存在系统变化(图 1). 正常柯 石英榴辉岩中磷灰石明显高于其他两类岩石的 $\mathrm{Cl}$ 含 量, 反映流体盐度在超高压条件下比高压条件下要 高. 这个特征表明, 大陆地壳岩石在俯冲过程中发生 水化反应导致岩石向干化方向发展, 即随着俯冲

引用格式: 刘景波, 张灵敏, 陈意, 等. 大别-苏鲁造山带超高压榴辉岩和脉体磷灰石含氯特征与变质流体演化. 科学通报, 2013, 58: 2165-2168 Liu J B, Zhang L M, Chen Y, et al. Chlorine contents in apatites of eclogites and hosted veins from the Dabie-Sulu UHP belt: Implication for fluid evolution in the process of metamorphism. Chin Sci Bull, 2013, 58: 2165-2168, doi: 10.1360/972013-578 


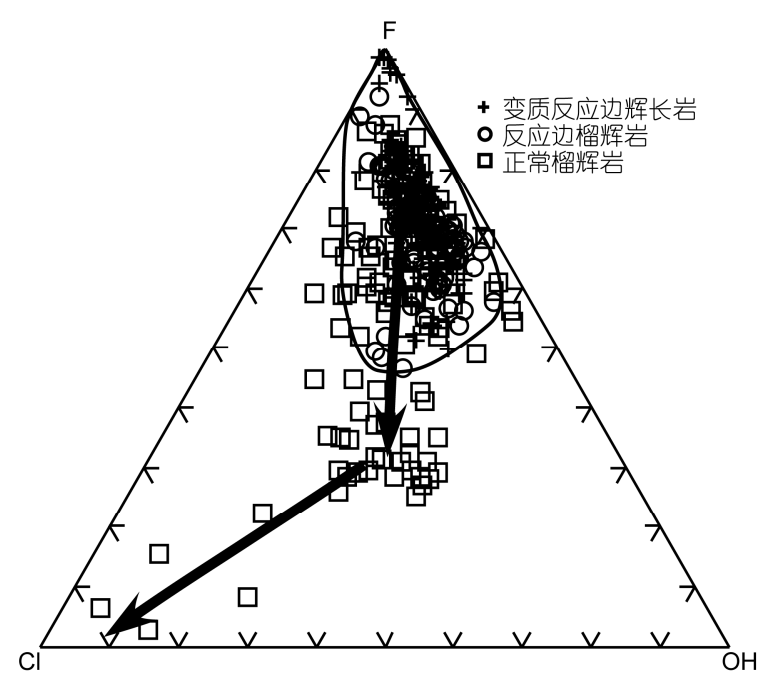

图 1 仰口变质反应边辉长岩、反应边榴辉岩和正常榴辉岩 中磷灰石的成分 ${ }^{[13]}$

箭头表示了磷灰石沿进变质方向的成分变化

深度增加, 陆壳流体会逐步被一些特定的水化变质 反应所吸收, 使得超高压变质的陆壳岩石向无水方 向发展.

对折返退变质过程中形成的产物，包括多硅白 云母石英脉、角闪石反应边、后成合晶反应中形成的 磷灰石也进行了系统挥发分成分研究(图 2), 折返过 程中随压力降低, 磷灰石氯含量降低, 反映出岩石在 折返过程中流体含量增加, 相应的流体盐度降低. 究 其原因可能是深俯冲大陆地壳折返过程中, 超高压 岩石中的多硅白云母发生分解, 产生流体并释放到 岩石之中, 使得大陆地壳朝着富流体方向发展 ${ }^{[13,14]}$.

\section{2 大别-苏鲁其他地区榴辉岩和高压脉体中 的磷灰石}

苏鲁超高压变质带中的露头包括池庄、青龙山和 毛北. 池庄榴辉岩中磷灰石氯含量为 $0.01 \%$ 2.34\% (质量分数, 下同), 榴辉岩中石英脉、富绿辉石-石英脉 和富多硅白云母-石英脉中磷灰石氯含量为 $0.0 \sim 0.2 \%$, 绿辉石 - 䵢帘石-蓝晶石-石英脉中磷灰石氯含量为 0.1\% 1.68\%. 青龙山榴辉岩中磷灰石氯含量为 $0.03 \%$ $0.35 \%$. 毛北浅色榴辉岩磷灰石氯含量为 $0.01 \%$ $0.18 \%$, 深色榴辉岩为 $0.2 \% \sim 0.45 \%$, 蓝晶石-石英脉 为 $0.32 \% \sim 0.36 \%$, 绿辉石脉为 $0.04 \% \sim 0.28 \%$, 黝帘石石英脉为 $0.0 \sim 0.1 \%{ }^{[15]}$.

大别造山带的露头包括碧溪岭、毛屋、朱家冲和

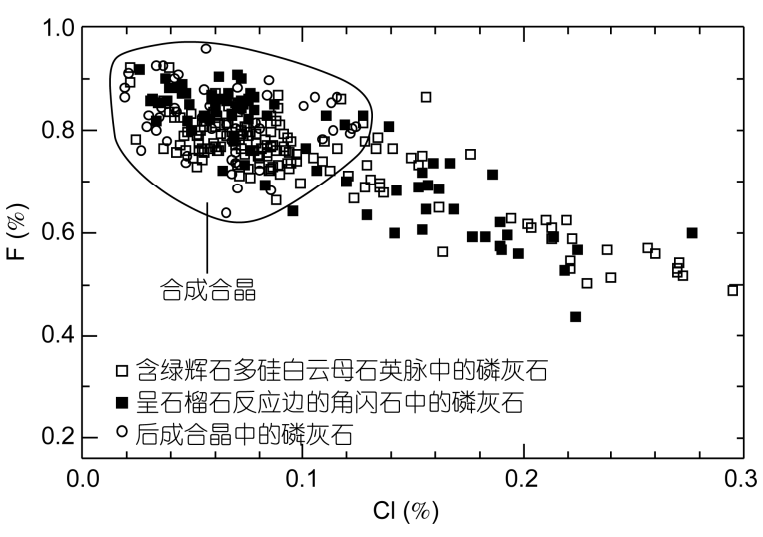

图 2 仰口榴辉岩类退变质磷灰石的成分特征 ${ }^{[14]}$

花凉亭. 碧溪岭浅色榴辉岩与深色榴辉岩中的磷灰 石氯含量范围相似，在 $0.1 \% \sim 1.45 \%$; 退变榴辉岩的 磷灰石氯含量比较低 $(<0.2 \%)$; 榴辉岩中绿辉石脉和 蓝晶石一石英脉的磷灰石氯含量为 $0.22 \% \sim 0.91 \%$. 花 凉亭榴辉岩及其中绿帘石脉、绿帘石-石英脉和绿帘 石-滑石-蓝晶石-石英脉中的磷灰石氯含量都很低 $(<0.3 \%)$. 朱家冲榴辉岩和蓝晶石-多硅白云母-石英 脉中磷灰石氯含量非常低 $(<0.05 \%)^{[15]}$. 毛屋各类基 性-超基性岩中的磷灰石氯含量都很高, 其中榴辉岩 中的磷灰石氯含量为 $0.4 \%$ 2.7\%, 石榴二辉石岩中的 为 $1.1 \% \sim 1.9 \%$, 石榴方辉橄榄岩中的为 $0.1 \% \sim 7 \%$, 退 变质的滑石透闪石岩中的为 $0.4 \% \sim 0.8 \%$.

\section{3 磷灰石成分指示的流体成分特征}

榴辉岩中磷灰石氯含量特征指示高压-超高压变 质过程中存在广泛的盐水流体活动. 磷灰石的 $X_{\mathrm{FAp}}$ / $X_{\mathrm{OHAp}}$ 和 $X_{\mathrm{ClAp}} / X_{\mathrm{OHAp}}$ 比值指示了岩石中流体成分的差 异 ${ }^{[15]}$. 池庄、碧溪岭和毛屋岩石中流体的盐度很高, 氟含量很低. 毛北和青龙山岩石中与磷灰石平衡的 流体具有高的氟含量和中等盐度. 朱家冲和花凉亭 榴辉岩中流体的氟含量中等, 盐度很低. 各类脉体中 磷灰石组分反映出成脉流体成分存在差异. 池庄的 绿辉石-䵢帘石-蓝晶石-石英脉磷灰石组分指示流体 盐度变化范围较大; 其他 3 类脉体以及毛北的脉体中 的磷灰石组分代表的流体盐度较低. 碧溪岭的两类 脉体中磷灰石对应的流体氟含量均很低, 盐度较高. 花凉亭的 3 类脉体磷灰石对应的流体氟含量低、盐 度低.

榴辉岩和脉体中磷灰石 $X_{\mathrm{CIAp}} / X_{\mathrm{OHAp}}$ 比值与变化 反映出与磷灰石平衡的流体盐度与变化 ${ }^{[15]}$. 榴辉岩 
变质过程中的流体是盐水流体, 盐度变化很大. 一方 面在一个标本上就显示了大的盐度变化, 表明变质 过程流体的盐度是变化的. 另一方面, 不同露头的比 值差别很大, 表明变质地壳不同区域流体的盐度也 是有差别的.

我们研究的典型榴辉岩露头中, 一些榴辉岩和 脉体已有流体包裹体盐度的研究结果, 包括青龙山 榴辉岩 ${ }^{[16]}$ 、池庄绿辉石-䵢帘石-蓝晶石-石英脉 ${ }^{[17,18] 、}$ 碧溪岭榴辉岩 ${ }^{[8]}$ 、朱家冲榴辉岩中多硅白云母-蓝晶 石-石英脉和䵢窝石-石英脉 ${ }^{[19]}$. 把我们测定的磷灰石 $X_{\mathrm{ClAp}} / X_{\mathrm{OHAP}}$ 比值与相应榴辉岩和脉体中不同期次流 体包裹体盐度对比, 形成了磷灰石 $X_{\mathrm{ClAp}} / X_{\mathrm{OHAp}}$ 比值 与流体包裹体盐度之间的相关关系(图 3). 从图 3 可 以看出, 磷灰石 $X_{\mathrm{ClAp}} / X_{\mathrm{OHAp}}$ 比值与流体包裹体盐度 呈很好的线性正相关关系. 榴辉岩和脉体磷灰石 $X_{\mathrm{ClAp}} / X_{\mathrm{OHAp}}$ 比值在 $0 \sim 0.35$ 所对应的流体包裹体盐度 约为 $0 \sim 40 \% \mathrm{NaCl}$ 当量. 根据这一关系, 可以通过磷 灰石的 $X_{\mathrm{ClAp}} / X_{\mathrm{OHAp}}$ 比值来估计与磷灰石平衡的流体 盐度.

总体来看，不同盐度的流体在变质过程中普遍 存在, 可能与大陆地壳俯冲/折返过程中岩石的水化 和脱水反应有关. 前人对大别-苏鲁造山带超高压变 质岩矿物稳定同位素和水含量研究表明, 大陆地壳 俯冲和折返过程中流体活动具有内部缓冲性质, 即

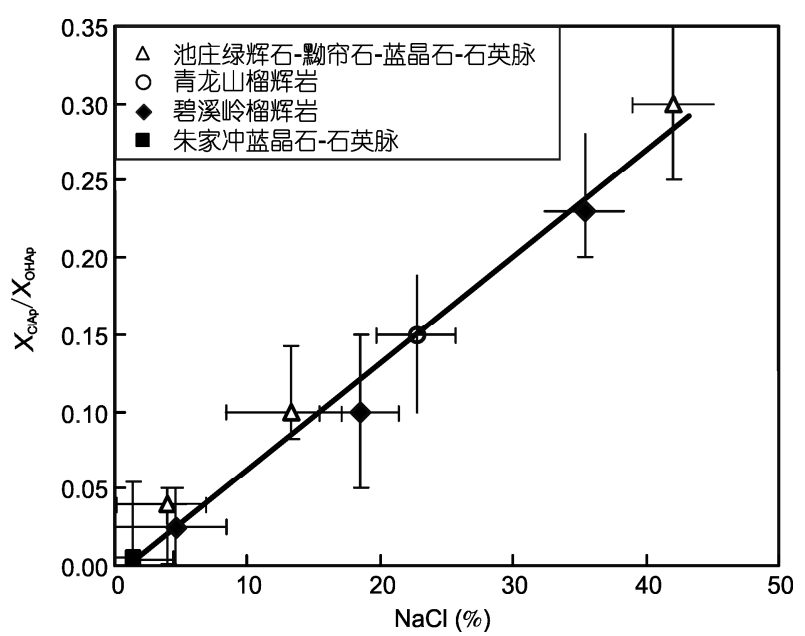

图 3 榴辉岩和脉体中磷灰石的 $\boldsymbol{X}_{\mathrm{CIAp}} / \boldsymbol{X}_{\mathrm{OHAp}}$ 与相应流体包裹 体盐度的关系 ${ }^{[15]}$

进变质和退变质流体均是来自俯冲/折返板片自 身 ${ }^{[20,21]}$. 仰口榴辉岩中磷灰石在进变质过程中显示 为氯含量增加, 退变质过程中氯含量降低, 可能与围 岩片麻岩多硅白云母的形成(水化反应)和分解(脱水 反应)相关, 前者反映了流体活动性减弱, 后者反映 流体活动性增加. 从这个关系来看, 磷灰石氯含量可 以用作度量大陆地壳俯冲和折返过程中板片内部流 体活动性的指标, 即高氯含量对应于低流体活动性, 而低氯含量对应于高流体活动性.

\section{参考文献}

1 Philippot P, Selverstone J. Trace-element-rich brines in eclogitic veins: Implications for fluid composition and transport during subduction. Contrib Mineral Petrol, 1991, 106: 417-430

2 Selverstone J, Franz G, Thomas S, et al. Fluid variability in 2 GPa eclogites as an indicator of fluid behaviour during subduction. Contrib Mineral Petrol, 1992, 112: 341-357

3 Philippot P, Chevallier P, Chopin C, et al. Fluid composition and evolution in coesite-bearing rocks (Dora-Maira massif, western Alps): Implication for element recycling during subduction. Contrib Mineral Petrol, 1995, 121: 29-44

4 Scambelluri M, Pennacchioni G, Philippot P. Salt-rich aqueous fluids formed during eclogitization of metabasites in the Alpine continental crust (Austroalpine Mt. Emilius unit, Italian western Alps). Lithos, 1998, 43: 151-167

5 Glassley W E. Elemental composition of concentrated brines in subduction zones and the deep continental crust. Precambrian Res, 2001, 105: 371-383

6 Scambelluri M, Philippot P. Deep fluids in subduction zones. Lithos, 2001, 55: 213-227

7 Svensen H, Jamteit B, Banks D A, et al. Halogen contents of eclogite facies fluid inclusions and minerals: Caledonides, western Norway. J Metamorph Geol, 2001, 19: 165-178

8 Xiao Y L, Hoefs J, van den Kerkhof A M, et al. Fluid history of UHP metamorphism in the Dabie Shan, China: A fluid inclusion and oxygen isotope study on the coesite-bearing eclogite from Bixiling. Contrib Mineral Petrol, 2000, 139: 1-16

9 Xiao Y L, Hoefs J, van den Kerkhof A M, et al. Fluid evolution during HP and UHP metamorphism in Dabie Shan, China: Constraints from mineral chemistry, fluid inclusions and stable isotopes. J Petrol, 2002, 43: 1505-1527 
10 Fu B, Touret J L R, Zheng Y F. Fluid inclusions in coesite-bearing eclogites and jadeite quartzites at Schuanghe, Dabie Shan, China. J Metamorph Geol, 2001, 19: 531-548

11 Fu B, Zheng Y F, Touret J L R. Petrological, isotopic and fluid inclusion studies of eclogites from Sujiahe, NW Dabie Shan (China). Chem Geol, 2002, 187: 107-128

12 Newton R C, Aranovich L Y, Hansen E C, et al. Hypersaline fluids in Precambrian deep-crustal metamorphism. Precambrian Res, 1998, 91: 41-63

13 Liu J B, Zhang L M, Mao Q, et al. Increasing chlorinity in fluids along the prograde metamorphic path: Evidence from apatite from Yangkou eclogite, Sulu, China. In: Dobrzhinetskaya L F, Faryad S W, Wallis S, et al., eds. Ultrahigh-Pressure Metamorphism: 25 Years After the Discovery of Coesite and Diamond. London: Elsevier, 2011. 213-242

14 Liu J B, Liu W Y, Ye K, et al. Chlorine-rich amphibole in Yangkou eclogite, Sulu ultrahigh-pressure metamorphic terrane, China. Eur J Miner, 2009, 21: 1265-1285

15 张灵敏, 刘景波, 程南飞, 等. 大别-苏鲁高压超高压带榴辉岩和脉体中磷灰石 $\mathrm{Cl}$ 含量和流体盐度关系的研究. 岩石学报, 2013, 29: $1525-1539$

16 翟伟，孙晓明，徐莉，等. 苏北青龙山超高压变质榴辉岩流体包裹体特征与流体演化. 岩石学报, 2005, 21: 482-488

17 沈昆，张泽明，石超，等. 江苏东海榴辉岩相变质脉体的流体包裹体研究. 岩石学报, 2008, 24: 1987-2002

18 Zhang Z M, Shen K, Sun W D, et al. Fluids in deeply subducted continental crust: Petrology, mineral chemistry and fluid inclusion of UHP metamorphic veins from the Sulu orogen, eastern China. Geochim Cosmochim Acta, 2008, 72: 3200-3228

19 Franz L, Romer R L, Klemd R, et al. Eclogite-facies quartz veins within metabasites of the Dabie Shan (eastern China): Pressure-temperature-time-deformation path, composition of the fluid phase and fluid flow during exhumation of high-pressure rocks. Contrib Mineral Petrol, 2001, 141: 322-346

20 Zheng Y F. Fluid regime in continental subduction zones: Petrological insights from ultrahigh-pressure metamorphic rocks. J Geol Soc, 2009, 166: 763-782

21 Zheng Y F. Metamorphic chemical geodynamics in continental subduction zones. Chem Geol, 2012, 328: 5-48 